\title{
Interplay between many body effects and Coulomb screening in the optical bandgap of atomically thin $\mathrm{MoS}_{2}$
}

Youngsin Park, †a Sang Wook Han, †b Christopher C. S. Chan,c Benjamin P. L. Reid,c Robert A. Taylor, ${ }^{*}$ c Nammi Kim,d Seung W. Lee,f Yongcheol Joe, Hyunsik Im*e and Kwang S. Kim*a

a. Department of Chemstry and Physics, School of Natural Science, Ulsan National Institute of Science and Technology, Ulsan 44919, Korea. E-mail: kimks@unist.ac.kr

b. Department of Physics and Energy Harvest \& Storage Research Center, University of Ulsan, Ulsan 44610, Korea.

c. $\quad$ Clarendon Laboratory, Department of Physics, University of Oxford, Oxford, OX1 3PU, UK. Email: robert.taylor@physics.ox.ac.uk

d. Department of Physics, Soongsil University, Seoul 06978, Korea.

e. Division of Physics and Semiconductor Science, Dongguk University, Seoul 04620, Korea. Email: hyunsik7@dongguk.edu

$\dagger$ These authors contributed equally to this work.

Due to its unique layer-number dependent electronic band structure and strong excitonic features, atomically thin $\mathrm{MoS}_{2}$ is an ideal 2D system where intriguing photoexcited-carrier-induced phenomena can be detected in excitonic luminescence. We perform micro-photoluminescence (PL) measurements and observe that the PL peak redshifts nonlinearly in mono- and bi- layer $\mathrm{MoS}_{2}$ as the excitation power is increased. The excited carrier-induced optical bandgap shrinkage is found to be proportional to $n^{4 / 3}$, where $n$ is optically-induced free carrier density. The large exponent value of $4 / 3$ is explicitly distinguished from a typical value of $1 / 3$ in various semiconductor quantum well systems. The peculiar $n^{4 / 3}$ dependent optical bandgap redshift may be due to the interplay between bandgap renormalization and reduced exciton binding energy.

\section{Introduction}

Single-layer $\mathrm{MoS}_{2}$ is a direct gap semiconductor with a bandgap of $1.8-1.9 \mathrm{eV}$ in a two-dimensional hexagonal Brillouin zone whereas bulk $\mathrm{MoS}_{2}$ is an indirect semiconductor with a band gap of $\sim 1.2 \mathrm{eV}$. ${ }^{1,2}$ Ultra-thin $\mathrm{MoS}_{2}$ layers with a thickness up to a few monolayers exhibit layer dependent crystal symmetry and a dramatic change in electronic dispersion. ${ }^{3,4}$ These materials have attracted interest due to their rich and intriguing optical and electrical properties. Atomically-thin $\mathrm{MoS}_{2}$ layers are perfect for nanooptoelectronic applications due to their semiconducting nature, ${ }^{5,7}$ excellent thermal stability, and absence of dangling bonds. ${ }^{8,9}$ Single-layer $\mathrm{MoS}_{2}$-based phototransistors and sensors have been demonstrated recently. ${ }^{10-12}$

The bandgap $\left(E_{\mathrm{g}}\right)$ is the most important parameter in the understanding of the optical properties of twodimensional (2D) semiconductors. Its value can change depending on external parameters such as temperature, pressure, and strain that can fundamentally alter the complex interactions between carriers and the lattice. ${ }^{13}$ As temperature $(T)$ decreases, the optical bandgap increases due to lattice dilation and electron-lattice interactions, which in turn causes a decrease in the potential seen in electrons in 2D semiconductors. On the other hand, bandgap renormalization (BGR) induced by many-body effects might occur in 2D semiconductors, resulting in a reduction of the bandgap. The BGR effect has been demonstrated in diverse semiconductor quantum wells (QWs). ${ }^{14-19}$ Bandgap shift $\left(\Delta_{\mathrm{BGR}}\right)$ in semiconductor quantum well (QW) systems has been found to be proportional to the density of charged carriers $(n), \Delta_{\mathrm{BGR}}$ $\propto n^{\alpha}$ where the exponent $\alpha$ is generally known to be close to $1 / 3 .{ }^{15,20-23}$ Another key fundamental factor for the understanding of the excitonic transition energy (optical bandgap) in low-dimensional semiconductor systems is the exciton binding energy. For 2D systems, suppression of effective Coulomb screening has been discussed ${ }^{24,25}$ and a large exciton binding energy of $>500 \mathrm{meV}$ has been predicted in monolayer $\mathrm{MoS}_{2}{ }^{26,27}$ 
In this study, we report the effect of photo-excited carriers on the optical bandgap in mono-(1L) and bilayer (2L) $\mathrm{MoS}_{2}$. We have measured excitonic spectra as a function of excitation power. We have observed the effect of photo-excited carrier densities on the excitonic spectra indicating the presence of many body effects and induced bandgap renormalization. We have found the following relationship in the low plasma density regime: $\Delta_{\mathrm{BGR}} \propto n^{4 / 3}$. A comparison between experimental and theoretical results reveals that the competition between bandgap renormalization and a lowering of the exciton binding energy due to Coulomb screening causes the large exponent value of 4/3.

\section{Experimental}

$1 \mathrm{~L}$ and $2 \mathrm{~L} \mathrm{MoS}_{2}$ flakes were prepared on a Si substrate covered with $285 \mathrm{~nm}$-thick-SiO${ }_{2}$ by mechanically exfoliating a bulk $2 \mathrm{H}-\mathrm{MoS}_{2}$ crystal. The optical luminescence properties were investigated by conducting micro-photoluminescence ( $\mu$-PL) measurements. The sample was mounted in a continuous-flow helium cryostat with temperature accurately controlled from $4.2 \mathrm{~K}$ to room temperature. A CW solid-state laser operating at a wavelength of $532 \mathrm{~nm}$ was used to excite the $\mathrm{MoS}_{2}$ flakes. The laser beam was focused onto the surface of the sample with a spot size of $\sim 0.8 \mu \mathrm{m}^{2}$. A $100 \times$ objective was held above the cryostat to focus the incident laser beam and collect the resulting luminescence. The luminescence was then directed to a spectrometer with a spectral resolution of $700 \mu \mathrm{eV}$.

\section{Results and discussion}

Figure 1a shows an optical microscope image of a sample $\mathrm{MoS}_{2}$ flake transferred onto the $\mathrm{SiO}_{2} / \mathrm{Si}$ substrate. The brown-coloured background represents the $\mathrm{SiO}_{2} / \mathrm{Si}$ substrate and the central regions with two different contrasts correspond to $\mathrm{MoS}_{2}$ layers with different thicknesses. The upper semi-transparent and lower dark regions correspond to the $1 \mathrm{~L}$ and $2 \mathrm{~L} \mathrm{MoS}_{2}$, respectively. The number of $\mathrm{MoS}_{2}$ layers was determined with Raman spectroscopy measurements at two different contrast regions as shown in Fig. $1 \mathrm{~b}$. Two prominent Raman bands (in-plane $\mathrm{E}_{2 \mathrm{~g}}{ }_{\mathrm{g}}$ and out-of-plane $\mathrm{A}_{1 \mathrm{~g}}$ modes) were observed. The frequency differences at the upper semi-transparent and bottom dark regions were $18.24 \mathrm{~cm}^{-1}$ and $21.10 \mathrm{~cm}^{-1}$, corresponding to the $1 \mathrm{~L}$ and $2 \mathrm{~L} \mathrm{MoS}_{2}$, respectively. ${ }^{28}$

Figure $1 \mathrm{c}$ shows the $\mu$-PL spectra obtained for $1 \mathrm{~L}-$ and $2 \mathrm{~L}-\mathrm{MoS}_{2}$ indicated by red and blue boxes, respectively, in Fig. 1a. For $1 \mathrm{~L}-\mathrm{MoS}_{2}$, two prominent PL peaks were present in the spectrum at $1.78 \mathrm{eV}$ (denoted as LE) and $1.88 \mathrm{eV}$ (denoted as A). These two peaks are direct excitonic transitions at the $\mathrm{K}$ point of the Brillouin zone. ${ }^{1}$ They were assigned to the excitons bound to surface impurities/defects (DX) and free exciton (FX), respectively. The DX peak is attributed to excitons bound to surface impurities/defects. ${ }^{6,19,29,30}$ Since the low-energy DX peak was strongly suppressed in oxide-covered single-layer $\mathrm{MoS}_{2}{ }^{31}$ its reduced energy might be due to additional binding to surface adsorbents. The inset indicates a small signature for the split-off valence band, which is referred to as the B exciton, which can also be detected in the $1 \mathrm{~L}-\mathrm{MoS}_{2}$ sample. ${ }^{30,32}$ The A exciton peak intensity in our $2 \mathrm{~L} \mathrm{MoS}_{2}$ is larger than that in $1 \mathrm{~L} \mathrm{MoS}_{2}$. We confirmed this behaviour for different $1 \mathrm{~L}$ and $2 \mathrm{~L} \mathrm{MoS}$ flakes (Fig. S1). It is known that the PL spectrum and intensity of atomically thin $\mathrm{MoS}_{2}$ can be affected by defects and the substrate as well as by layer number. ${ }^{1,29,32-34}$ Thus, we presume that complex temperature-dependent interplay between these factors is linked to this observation. A very different PL behaviour was observed for the spectrum in the region of the $2 \mathrm{~L}-\mathrm{MoS}_{2}$. Only the FX peak was identified at around $1.88 \mathrm{eV}$. Band structure theory indicates that different excitonic effects in $1 \mathrm{~L}-$ and $2 \mathrm{~L}-\mathrm{MoS}_{2}$ (i.e., binding energies of $0.897 \mathrm{eV}$ for $1 \mathrm{~L}-\mathrm{MoS}_{2}$ and $0.424 \mathrm{eV}$ for $2 \mathrm{~L}-\mathrm{MoS}_{2}$ ) should be considered in order to understand the optical transitions. ${ }^{35}$ The physical nature of the $\mathrm{MoS}_{2} / \mathrm{SiO}_{2}$ interface might cause different strains in the $1 \mathrm{~L}-$ and $2 \mathrm{~L}-\mathrm{MoS}_{2}{ }^{19,36} \mathrm{In}$ addition, many groups have demonstrated that $1 \mathrm{~L}-\mathrm{MoS}_{2}$ suspended from the substrate or encapsulated by dielectric materials has different PL emission characteristics compared to a monolayer that only has contact with a $\mathrm{SiO}_{2} / \mathrm{Si}$ substrate. ${ }^{1.31}$ Given these observations, PL spectra in atomically thin $\mathrm{MoS}_{2}$ can be influenced by their intrinsic electronic band structures and surrounding environment. The reproducibility of this behaviour throughout the entire $\mathrm{MoS}_{2}$ flake was verified by taking PL intensity 
mapping measurements at the $\mathrm{LE}$ and A peak energies of $1.78 \mathrm{eV}$ and $1.88 \mathrm{eV}$, respectively, as shown in Figures $1 \mathrm{~d}$ and 1e. Comparison of PL confocal mapping with an optical image shows that the $1 \mathrm{~L}$ and $2 \mathrm{~L}$ regions have very different emission characteristics.

Before showing the experimental data, we first describe how the photo-excited carriers affect the excitonic transition energy. In the low excitation power regime where the thermal heating effect is insignificant, the major contributions to the shift in the excitonic PL in low dimensional-semiconductor systems are determined by the interplay between the BGR effect (due to electron and hole self-energy corrections) and screening-induced excitonic binding weakness. ${ }^{4,37}$ The former process (the BGR effect) causes a redshift in the optical bandgap, and it competes with the latter, which causes a blueshift (see Fig. 2 ). As the photo-excited carrier density $(n)$ increases, the excitonic transition energy ( $E_{\text {opt }}$ in Fig. $\left.2 \mathrm{~b}\right)$ shifts due to the self-energy corrections and Coulomb-screening whose magnitudes change nonlinearly with respect to $n .^{4}$

The excitation power-dependent micro-PL spectra of $1 \mathrm{~L}-$ and $2 \mathrm{~L}-\mathrm{MoS}_{2}$ are shown in Figures $3 \mathrm{a}$ and $3 b$, respectively. Note that the intensity of the LE transition in $1 \mathrm{~L}-\mathrm{MoS}_{2}$ is very weak, and this is due to the non-uniform adsorbent condition of the $1 \mathrm{~L}-\mathrm{MoS}_{2}$ flake. As the excitation power increases, the Aexciton peak in both $1 \mathrm{~L}-$ and $2 \mathrm{~L}-\mathrm{MoS}_{2}$ shifts monotonically towards the lower energy side. As the temperature increases in the region below $100 \mathrm{~K}$, the PL peak's height decreases dramatically and its position remains almost unchanged (Fig. S2). However, the observed PL peak's height increases linearly with excitation power (see Fig. 4a). Thus it is reasonable to assume that the redshift of the powerdependent PL peak is unrelated to the rise in the effective temperature induced by the excitation power. Mak et al and Kummell et al reported the observation of trions in $1 \mathrm{~L}-$ and $2 \mathrm{~L}-\mathrm{MoS}_{2} \cdot{ }^{30,38}$ However, considering the very symmetric shape of the PL spectra of our $1 \mathrm{~L}-$ and $2 \mathrm{~L}-\mathrm{MoS}_{2}$, which are well fitted by a single-Gaussian curve, trions seem not to contribute to the observed PL spectra in our sample.

Variation in the PL intensity of the A-exciton peak with excitation power $\left(P_{\mathrm{ex}}\right)$ is shown in Figure 4a. PL spectra were measured in the photo-excited quasi-equilibrium regime. The peak's intensity $\left(I_{\text {peak }}\right)$ increases linearly with increasing excitation power, $I_{\text {peak }} \propto P_{e x}$. In the quasi-equilibrium region, it is reasonable to presume that $I_{\text {peak }}$ is linearly proportional to the density of the excitons $\left(n_{\mathrm{ex}}\right)$. Thus, it is reasonable to presume $n_{\mathrm{ex}} \propto P_{\text {ex }}$. Figure 4(b) shows the PL peak's redshift ( $\Delta E_{\mathrm{opt}}$ ) relative to PL peaks measured at the lowest excitation powers used $\left(3.8\right.$ and $3.2 \mathrm{~kW} / \mathrm{cm}^{2}$ for $1 \mathrm{~L}-$ and $2 \mathrm{~L}-\mathrm{MoS}_{2}$, respectively). As $P_{e x}$ increased, $\Delta E_{\text {opt }}$ also increased. Their relation was found to be $\Delta E_{\mathrm{opt}} \propto P_{\mathrm{ex}}{ }^{2 / 3}$ for both samples. For comparison, the $\Delta E_{\text {opt }} \propto P_{\mathrm{ex}}{ }^{1 / 3}$ relation is indicated with a red solid line. However, in a higher excitation power (or higher plasma $e-h$ density) regime, the power-dependent exciton peak's energy deviated from the $\Delta E_{\mathrm{opt}} \propto P_{\mathrm{ex}}{ }^{2 / 3}$ behaviour and red-shifted with a smaller exponent, indicating that the exciton binding strength might have been weakened by an increased effective Coulomb screening. The observed exciton peak's redshift implies that the Coulomb-driven quasiparticle BGR effect was dominant over the freecarrier screening induced exciton binding energy lowering. A similar narrowing in the optical bandgap in monolayer semiconductor systems has been recently demonstrated experimentally and theoretically. $4,26,39$ To better understand the results of our experiment, we need to know the physics of the equilibrium between the excitons and the dense electron-hole $(e-h)$ plasma. The density ratio is sensitively determined by both lattice temperature and exciton binding energy. For quasi $2 \mathrm{D}$ intrinsic semiconductors in the low plasma density region (or in the low excitation power region), the Saha equation might be adopted from plasma physics to describe the density ratio as follows: ${ }^{40-42}$

$$
n_{\mathrm{e}} \times n_{\mathrm{h}} / n_{\mathrm{ex}} \propto k T \times \exp \left(-E_{\mathrm{b}} / k T\right)
$$

where $E_{\mathrm{b}}$ is the binding energy of the exciton and $n_{\mathrm{e}, \mathrm{h}}$ represents the density of the conducting electrons and holes. $k T$ is the effective thermal energy of $2 \mathrm{D} \mathrm{MoS}$. In the presence of screening of free carriers $(n$ $\equiv n_{\mathrm{e}}=n_{\mathrm{h}}$ ), the ratio $E_{\mathrm{b}} / k T$ value changes with increasing excitation power (note that the binding energy $E_{\mathrm{b}}$ decreases due to the screening of the increased plasma density). Since $E_{\mathrm{b}}>k T$ in the Saha equation remains valid and the density of the free electrons $n_{\mathrm{e}}$ in the conduction band increases in proportion to the square root of the exciton density $n_{\mathrm{ex}}$, the following relation is obtained: $n \propto n_{\mathrm{ex}}{ }^{1 / 2}$. The behaviours of 
$\Delta E_{\mathrm{opt}} \propto P_{\mathrm{ex}}^{2 / 3}$ and $P_{\mathrm{ex}} \propto n_{\mathrm{ex}}$ lead to following relation: $\Delta E_{\mathrm{opt}} \propto n^{1.33}$. Figure 5a presents the measured $\Delta E_{\mathrm{opt}}$ on a logarithmic scale.

Steinhoff et al. have demonstrated theoretically the effects of excited carriers on the optical and electronic properties of $1 \mathrm{~L}-\mathrm{MoS}_{2}{ }^{4}$ Figure $5 \mathrm{~b}$ shows the calculated $\Delta E_{\text {opt }}$ (black solid line) for unstrained $1 \mathrm{~L}-\mathrm{MoS}_{2}$. As the carrier density $(n)$ increases, the bandgap renormalization $\left(\Delta E_{\mathrm{g}}\right)$ and the reduction of the binding energy $\left(\Delta E_{\mathrm{b}}\right)$ compete with each other and determine the exciton transition energy shift $\left(\Delta E_{\mathrm{opt}}\right)$. The theory predicts that as $n$ increases $\Delta E_{\text {opt }}$ increases non-monotonically following a power law of $\Delta E_{\text {opt }}$ $\propto n^{\gamma}$ where $\gamma$ decreases in a higher $n$ region. The BGR effect is dominant in the low $n$ region below $0.33 \times 10^{11} / \mathrm{cm}^{2}$, the Coulomb screening induced exciton binding energy reduction prevails in the high $n$ region $>2.2 \times 10^{12} / \mathrm{cm}^{2}$. We determined the theoretical $\Delta E_{\text {opt }}(n)$ zone which best matched the measured experimental $\Delta E_{\mathrm{opt}}=\sim 8 \mathrm{meV}$ for the experimentally observed relation $\Delta E_{\mathrm{opt}} \propto n^{1.33}$. In the $n$ region between 0.5 and $1.1 \times 10^{12} / \mathrm{cm}^{2}$, the theoretical $\Delta E_{\text {opt }}$ satisfies the experimentally observed $\Delta E_{\mathrm{opt}} \propto n^{1.33}$ behaviour. The experimental data overlap with the theoretical results where the standard deviation between them is minimized. There were no fitting parameters for this comparison. The $n$ region between $\sim 6 \times 10^{11} / \mathrm{cm}^{2}$ and $\sim 9 \times 10^{11} / \mathrm{cm}^{2}$ best satisfies the condition with the smallest standard deviation.

The $1 \mathrm{~L}$ - and $2 \mathrm{~L}-\mathrm{MoS}_{2}$ layers show a similar optical bandgap shift with increasing $P_{\text {ex }}$, suggesting that the interplay between the BGR effect and the exciton binding energy reduction plays a comparable role in determining the optical bandgap in $1 \mathrm{~L}-$ and $2 \mathrm{~L}-\mathrm{MoS}_{2}$. This is presumably due to the comparable effects of many-body interactions on the electronic structure near the $\mathrm{K}$ point in $1 \mathrm{~L}$ - and $2 \mathrm{~L}-\mathrm{MoS}_{2}$. Note that bandgap energy renormalization by many-body interactions seems not to depend strongly on the thickness of quasi-2D semiconductor quantum wells, ${ }^{14,15,18,20}$ because the binding energy of the $1 \mathrm{~L}$ - and $2 \mathrm{~L}$ is a few hundred meV while that of bulk is only a few tens of meV.

\section{Conclusions}

In summary, we investigated the effect of electron-electron interactions on the optical bandgap of monoand bi-layer $\mathrm{MoS}_{2}$ by measuring the excitation power-dependent PL characteristics. Two prominent direct excitonic PL peaks were observed for bound and free excitons. We demonstrated that photo-excited free carriers were able to modify the optical bandgap of these materials. It was found that the optical bandgap shift $\left(\Delta E_{\text {opt }}\right)$ increased proportional to a power of the free carrier density $(n)$ with a large exponent, $\Delta E_{\text {opt }}$ $\propto n^{1.33}$, in the low plasma density regime where many body effect-induced bandgap renormalization and exciton binding energy lowering competed with each other.

\section{Acknowledgements}

We would like to thank C. Gies and A. Steinhoff for providing their raw data in ref [4]. This research was supported by Basic Science Research Program and National Honor Scientist Program through the National Research Foundation of Korea (NRF) (Grant Nos. 2010-0020414, 2015R1D1A1A01058332, 2015R1A2A2A01004782, 2016R1A6A1A03012877 and 2015M2A2A6A02045252).

\section{Notes and references}

K. F. Mak, C. Lee, J. Hone, J. Shan and T. F. Heinz, Phys. Rev. Lett., 2010, 105, 136805.

G. L. Frey, S. Elani, M. Homyonfer, Y. Feldman, and R. Tenne, Phys. Rev. B, 1998, 57, 6666.

J. K. Ellis, M. J. Lucero, and G. E. Scuseria, Appl. Phys. Lett., 2011, 99, 261908.

A. Steinhoff, M. Rösner, F. Jahnke, T.O. Wehling, and C. Gies, Nano Lett., 2014, 14, 3743.

A. Splendiani, L. Sun, Y. Zhang, T. Li, J. Kim, C.-Y. Chim, G. Galli, and F. Wang, Nano Lett., 2010, 10, $1271-1275$.

T. Korn, S. Heydrich, M. Hirmer, J. Schmutzler, and C. Schuller, Appl. Phys. Lett., 2011, 99, 102109.

G. Eda, H. Yamaguchi, D. Voiry, T. Fujita, M. Chen, and M. Chhowalla, Nano Lett., 2011, 11, 5111-5116.

B. Radisavljevic, A. Radenovic, J. Brivio, V. Giacometti, and A. Kis, Nature Nanotech., 2011, 6, 147-150.

H. Wang, L. Yu, Y.-H. Lee, Y. Shi, A. Hsu, M. L. Chin, L.-J. Li, M. Dubey, J. Kong, and T. Palacios, Nano Lett., 2012, 12, 4674-4680. 
K. F. Mak, K. He, J. Shan, and T. F. Heinz, Nature Nanotech., 2012, 7, 494-498.

H. Zeng, J. Dai, Y. Wang, D. Xiao, and X. Cui, Nature Nanotech., 2012, 7, 490-493.

D. Xiao, G.-B. Liu, W. Feng, X. Xu, and W. Yao, Phys. Rev. Lett., 2012, 108, 196802.

P. Yu and M Cardona, Fundamentals of Semiconductors: Physics and Materials Properties 4th ed., Springer, 2010.

J. C. Ryan and T. L. Reinecke, Phys. Rev. B, 1993, 47, 9615.

D. A. Kleinman and R. C. Miller, Phys. Rev. B, 1985, 32, 2266-2272.

S. Schmitt-Rink, C. Ell, S. W. Koch, H. E. Schmidt, and H. Haug, Solid State Commun., 1984, 52, 123.

C. Ell and H. Haug, Phys. Status Solidi B, 1990, 159, 117.

S. Das Sarma, R. Jalabert, and S.-R. Eric Yang, Phys. Rev. B., 1989, 39, 5516.

Y. J. Wang, S. J. Xu, Q. Li, D. G. Zhao, and H. Yang, Appl. Phys. Lett., 2006, 88, 041903.

C. Delalande, G. Bastard, J. Orgonasi, J. A. Brum, H. W. Liu, M. Voos, G. Weimann, and W. Schlapp, Phys. Rev. Lett., 1987, 59, 2690 .

G. Tränkle, E. Lach, A. Forchel, F. Scholz, C. Ell, H. Haug, G. Weimann, G. Griffiths, H. Kroemer, and S. Subbanna, Phys. Rev. B, 1987, 36, 6712 .

X. Zhang, P. Kung, A. Saxler, D. Walker, and M. Razeghi, J. Appl. Phys., 1996, 80, 6544.

Q. Wu, R. D. Grober, D. Gammon, and D. S. Katzer, Phys. Rev. B, 2000, 62, 13022.

T. O. Wehling, E. Sasioglu, C. Friedrich, A. I. Lichtenstein, M. I. Katsnelson, and S. Blügel, Phys. Rev. Lett., 2011, 106,

23680 .

V. N. Kotov, B. Uchoa, V. M. Pereira, F. Guinea, and A. H. Castro Neto, Rev. Mod. Phys., 2012, 84, 1067.

D. Y. Qiu, F. H. da Jornada, and S. G. Louie, Phys. Rev. Lett., 2013, 111, 216805.

F. Hüser, T. Olsen, and K. S. Thygesen, Phys. Rev. B, 2013, 88, 245309.

C. Lee, H. Yan, L. E. Brus, T. F. Heinz, J. Hones, and S. Ryu, ACS Nano, 2010, 4, 2695.

D. Sercombe, S. Schwarz, O. Del Pozo-Zamudio, F. Liu, B. J. Robinson, E. A. Chekhovich, I. I. Tartakovskii, O. Kolosov, and A. I. Tartakovskii, Sci. Rep., 2013, 3, 3489.

T. Kummell, W. Quitsch, S. Matthis, T. Litwin, and G. Bacher, Phys. Rev. B, 2015, 91, 125305.

G. Plechinger, F. -X. Schrettenbrunner, J. Eroms, D. Weiss, C. Schuller, and T. Korn, Physica Status Solidi (RRL), 2012, 6, 126-128.

O. Salehzadeh, N. H. Tran, X. Liu, I. Shih and Z. Mi, Nano Lett., 2014, 14, 4125-4130.

H. Nan, Z. Wang, W. Wang, Z. Liang, Y. Lu, Q. Chen, D. He, P. Tan, F. Miao, X. Wang, J. Wang and Z. Ni, ACS nano, 2014, 8, 5738-5745.

W. Su, L. Jin, X. Qu, D. Huo and L. Yang, Phys. Chem. Chem. Phys., 2016, 18, 14001-14006

T. Cheiwchanchamnangij and W. R. L. Lambrecht, Phys. Rev. B, 2011, 85, 205302.

W. S. Yun, S. W. Han, S. C. Hong, I. G. Kim, and J. D. Lee, Phys. Rev. B, 2012, 85, 033305.

S. Das Sarma and D. W. Wang, Phys. Rev. Lett., 2000, 84, 2010-2013.

K. F. Mak, K. He, C. Lee, G. H. Lee, J. Hone, T. F. Heinz and J. Shan, Nature Mater., 2013, 12, $207-211$.

M. M. Ugeda, A. J. Bradley, S.-F. Shi, F. H. da Jornada, Y. Zhang, D. Y. Qiu, W. Ruan, S.-K. Mo, Z. Hussain, Z.-X. Shen, F. Wang, S. G. Louie, and M. F. Crommie, Nature Mater., 2014, 13, 1091.

D. S. Chemla, D. A. B. Miller, P. W. Smith, A. C. Gossard, and W. Wiegmann, IEEE J. Quantum Electron., 1984, 20, 265275.

R. T. Phillips, G. C. Nixon, T. Fujita, M. Y. Simmons, and D. A. Ritchie, Solid State Commun., 1996, 98, 287.

J. Szczytko, L. Kappei, J. Berney, F. Morier-Genoud, M. T.

Portella-Oberli, and B. Deveaud, Phys. Rev. Lett., 2004, 93,

137401. 
(a)

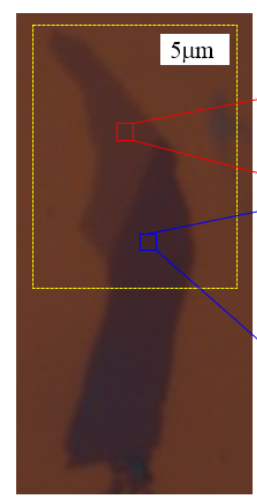

(b)

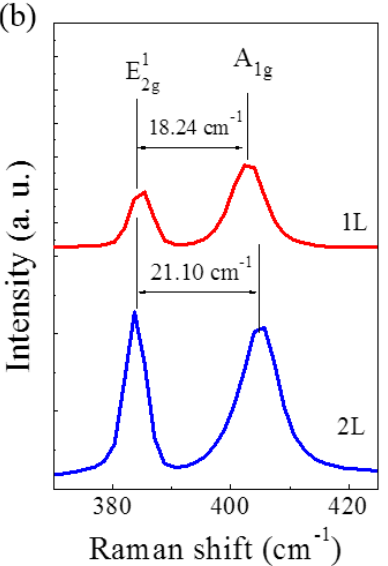

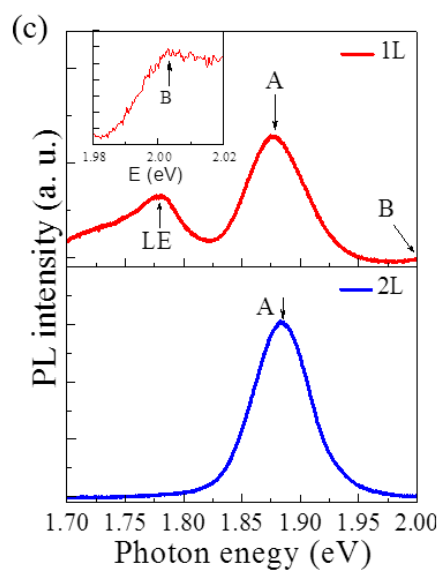

(d)

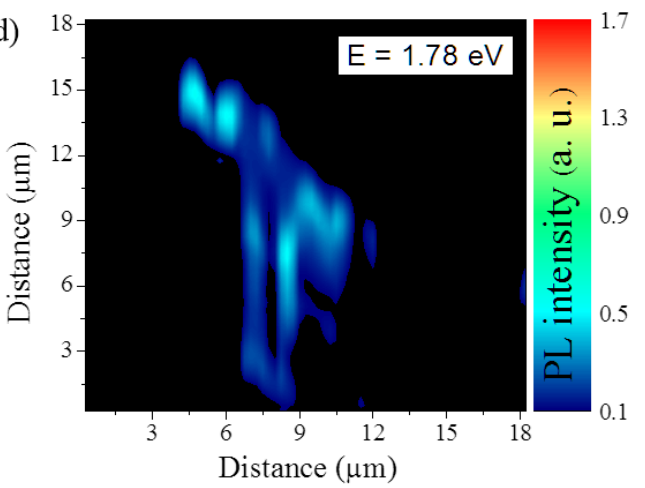

(e)

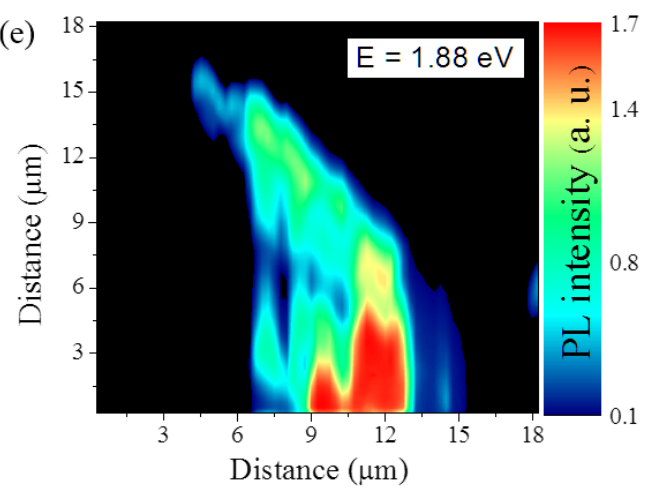

Fig. 1 (a) Optical microscopy image of $1 \mathrm{~L}$ and $2 \mathrm{~L} \mathrm{MoS}_{2}$ on a $\mathrm{SiO}_{2} / \mathrm{Si}$ substrate. In the schematic model, red and blue boxes correspond to $1 \mathrm{~L}$ and $2 \mathrm{~L}$ optical images, respectively. (b) Raman spectroscopy of $1 \mathrm{~L}-$ and $2 \mathrm{~L}-\mathrm{MoS}_{2}$ on a SiO $/ 2 / \mathrm{Si}$ substrate. (c) 4.2 K $\mu$-PL spectra taken at points corresponding to $1 \mathrm{~L}-$ and $2 \mathrm{~L}-\mathrm{MoS}_{2}$ (marked with red and blue boxes in (a), respectively). An excitation power of $128 \mathrm{~kW} / \mathrm{cm}^{2}$ was used. The inset shows the magnified PL spectrum of $1 \mathrm{~L}-\mathrm{MoS}_{2}$ in the high photon energy region. The small peak is referred to as B-exciton which is due to the splitting-off of the valence band. (d, e) PL intensity mapping images at different photon energies of $1.78 \mathrm{eV}$ and $1.88 \mathrm{eV}$ corresponding to LE and A peaks, respectively. An excitation power of $3.8 \mathrm{~kW} / \mathrm{cm}^{2}$ was used.

(a)

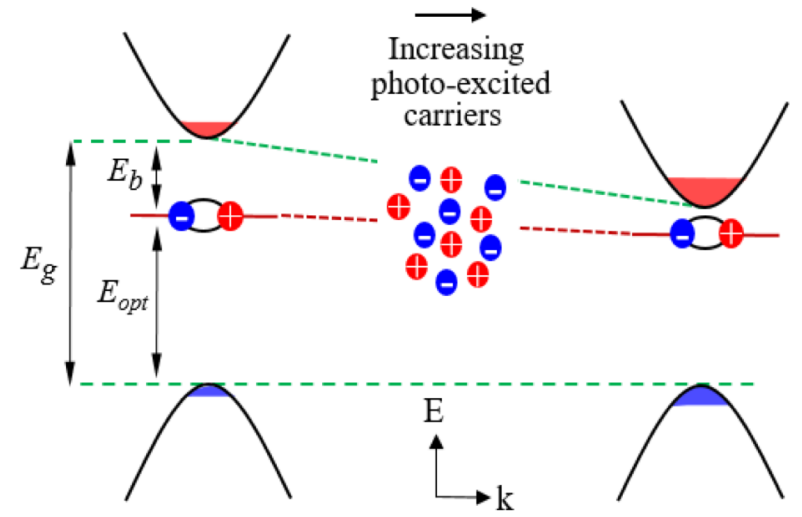

(b)

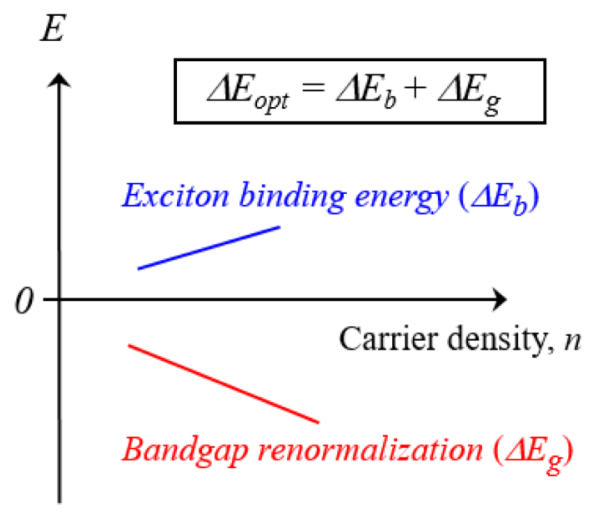

Fig. 2 (a) Illustration of the effect of photo-excited carriers on the bandgap $\left(E_{g}\right)$ and exciton binding energy $\left(E_{b}\right)$. The excitonic transition energy $\left(E_{\text {opt }}\right)$ is given by the relation of $E_{\text {opt }}=E_{g}-E_{b}$. (b) Schematic excitonic transition energy shift $\left(\Delta E_{\text {opt }}\right)$ due to two competing processes (not to scale), bandgap renormalization $\left(\Delta E_{g}\right)$ and exciton binding energy lowering $\left(\Delta E_{b}\right)$ with increasing photo-excited carrier density $(n)$. Positive and negative values of $\Delta E_{o p t}$ in the $y$-axis correspond to blue- and redshifts of the excitonic transition, respectively. Note that $\Delta E_{b}$ and $\Delta E_{g}$ are not linearly proportional to the charge density $n$. 
(a)

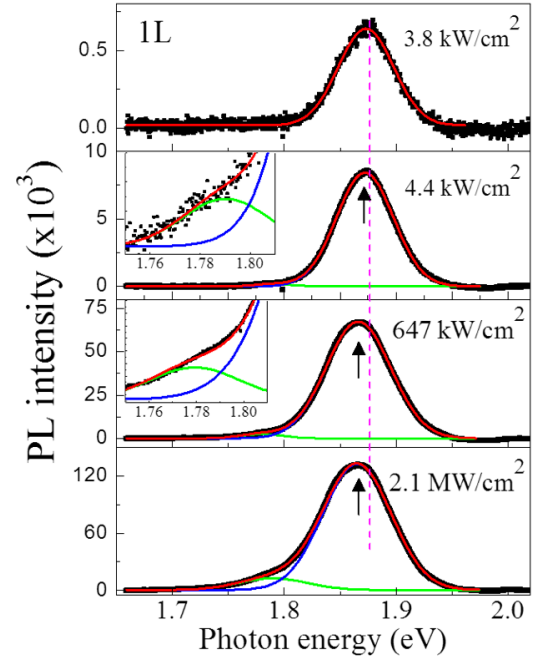

(b)

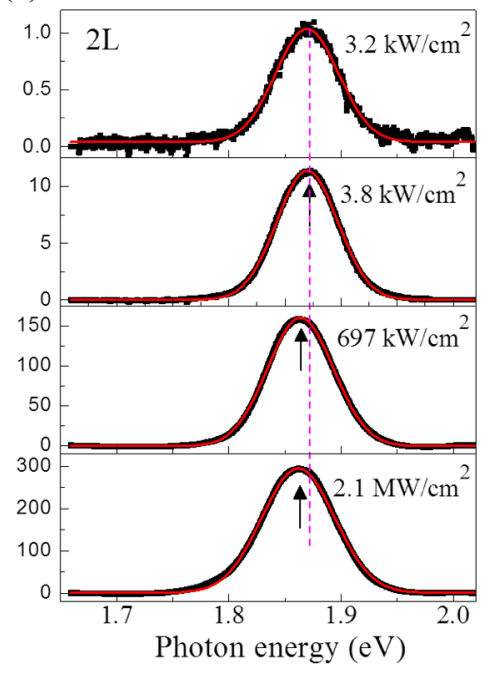

Fig. 3 (a,b) Representative excitation power-dependent micro-PL spectra measured at $4.2 \mathrm{~K}$ of the $1 \mathrm{~L}$ - and $2 \mathrm{~L}-\mathrm{MoS}_{2}$, respectively. All measured PL curves (black dots) were well fitted by using a Gaussian function. The insets depict the magnified PL spectra in the low photon energy region, together with fitted cur (blue lines for A, green lines for LE, and red lines for PL spectra reconstructed using the best fitting procedures) demonstrating the evolution of LE emission at high excitation powers. Vertical black arrows indicate peak positions.

(a)

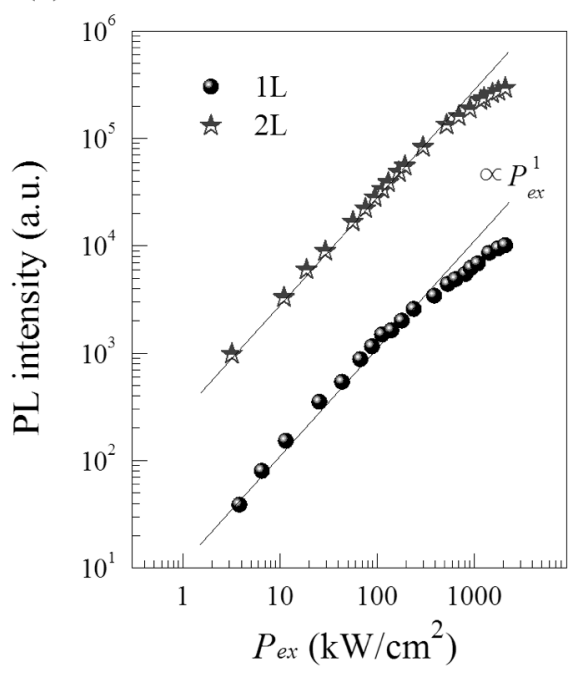

(b)

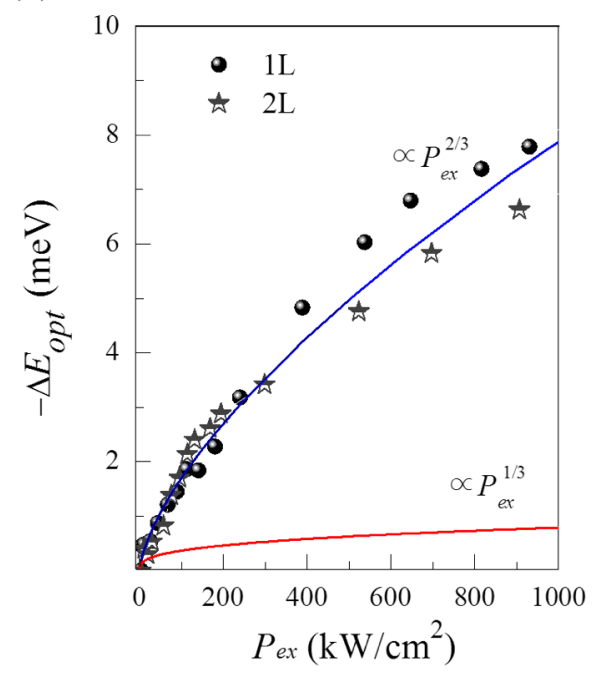

Fig. 4 (a) Intensity of the A-exciton peak as a function of excitation power at 4.2 K. (b) ] excitation power-dependent A exciton peak energy relative to the peak position of the PI powers of 3.8 (for $1 \mathrm{~L}-\mathrm{MoS}_{2}$ ) and $3.2 \mathrm{~kW} / \mathrm{cm}^{2}$ (for $2 \mathrm{~L}-\mathrm{MoS}_{2}$ ). The 1/3 and 2/3 power-law expression: $-\Delta E_{\text {opt }}=\mathrm{A} \times P_{e x}{ }^{\alpha}$ where $\mathrm{A}=8 \times 10^{-5}$. 
(a)

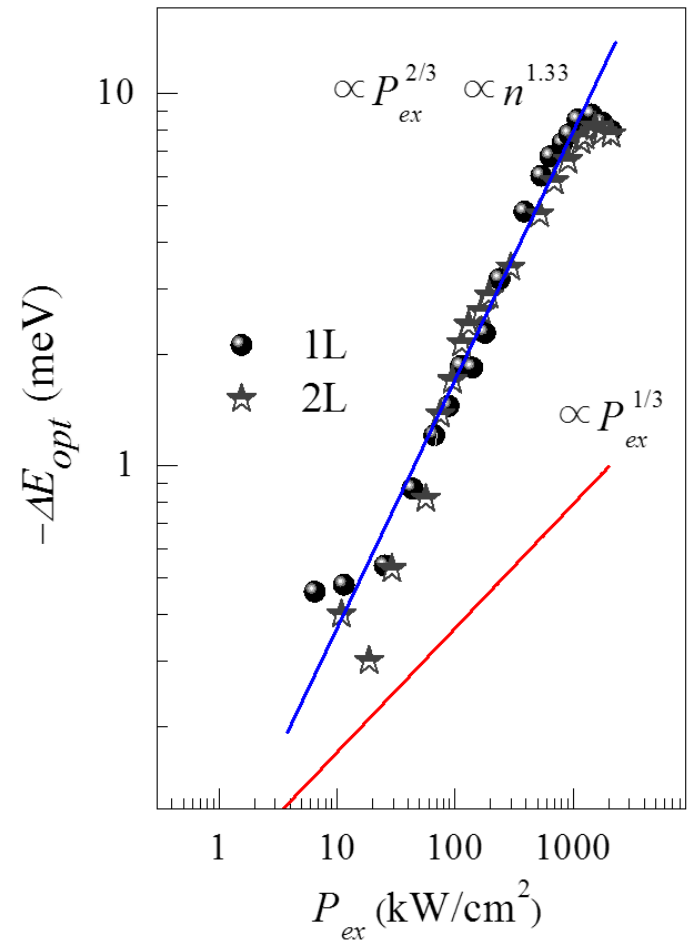

(b)

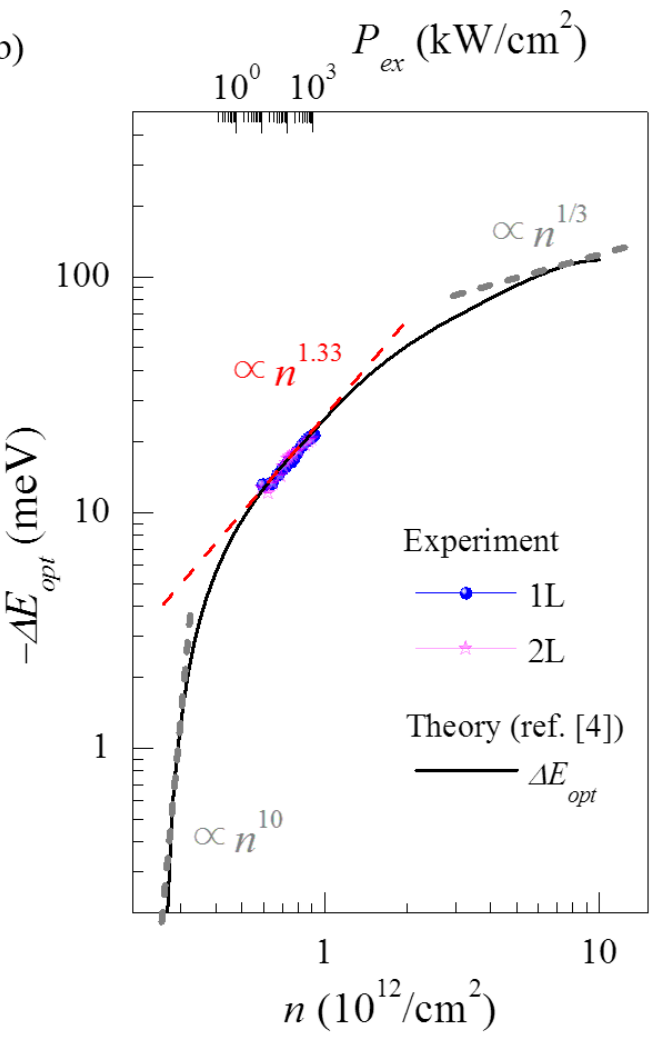

Fig. 5 (a) The redshift $\left(-\Delta E_{\text {opt }}\right.$ ) of the excitation power-dependent A exciton peak energy on a logarithmic scale. (b) Comparison of the theoretical $\Delta E_{\text {opt }}$ and the experimentally observed bandgap shift. For comparison, the experimental bandgap shift curve is shifted up in energy by $15 \mathrm{meV}$. The theoretical excitonic transition energy shift $\left(-\Delta E_{\text {opt }}\right)$ curve in unstrained $1 \mathrm{~L} \mathrm{MoS}_{2}$ is obtained from Ref. [4]. The theoretical $\Delta E_{\text {opt }}$ curve is determined by considering the competing phenomena, bandgap shrinkage $\left(\Delta E_{g}\right)$ and binding energy lowering $\left(\Delta E_{b}\right)$ of the A exciton transition: $\Delta E_{o p t}=\Delta E_{g}+\Delta E_{b}$ (see Fig. 2). The minus sign indicates that the exciton peak redshifts with increasing $n,\left(\left|\Delta E_{g}\right|>\left|\Delta E_{b}\right|\right)$. 